\title{
Enantiospecific Adsorption and Decomposition of D- and L-Asp Mixtures on $\mathrm{Cu}(643)^{R \& S}$
}

\author{
Soham Dutta ${ }^{a}$ and Andrew J. Gellman ${ }^{\star a b}$
}

\begin{abstract}
The study of molecular chirality is essential to understanding the fundamentals of enantiospecific chemical interactions that are ubiquitous in the biochemistry of life on Earth. At a molecular level, there is insufficient understanding of chiral recognition and enantiomer-enantiomer interaction (aggregation) of chiral molecules adsorbed on surfaces. Here, using enantiospecific isotopic labelling and surface sensitive techniques, we show that when the two enantiomers of chiral aspartic acid (Asp) are adsorbed on the naturally chiral $\mathrm{Cu}(643)^{\text {Res }}$ surfaces, they decompose enantiospecifically depending on the chirality of the surface. The non-linear kinetics of the surface decomposition mechanism amplifies the difference between the decomposition rate constants of the two adsorbed enantiomers resulting in highly enantiospecific decomposition rates. Further, we also demonstrate that Asp enantiomers aggregate homochirally on several chiral and achiral surfaces, amplifying the enantiomeric excess on the surface with respect to that in the gas phase, $\left|e e_{s}\right|>\left|e e_{g}\right|$. Our results show that it is possible to discern the enantiospecific behavior of a complex adsorbate such as Asp and shed light on molecular level enantiospecific interactions on surfaces. The enantiospecific isotope labelling methods discussed in this paper allow probing of both the qualitative features of the Asp decomposition mechanism on $\mathrm{Cu}(643)^{\text {ReS }}$ and quantitative aspects of the adsorption equilibria of enantiomer mixtures.
\end{abstract}

Keywords: Adsorption · Amino acids · Chirality · Mechanisms · Surfaces
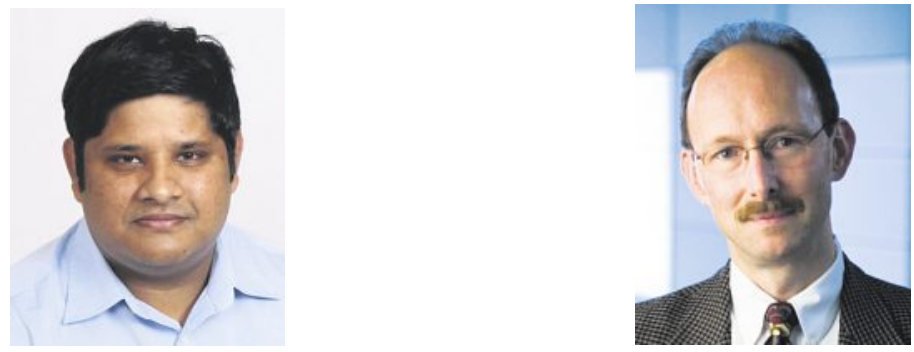

Soham Dutta is a $\mathrm{PhD}$ student in the Department of Chemical Engineering at Carnegie Mellon University in Pittsburgh, Pennsylvania, USA. He received his Bachelor of Chemical Engineering at Siddaganga Institute of Technology, Tumkur, India and his Master of Science in Chemical Engineering at Carnegie Mellon University. He is currently a member of the Catalysis and Surface Science laboratory and is studying decomposition and aggregation of chiral molecules on single crystal metal surfaces. Fundamental understanding of such phenomena has applications in enantiomer separation, enantioselective catalysis and supramolecular chirality.
Andrew Gellman is the Thomas Lord Professor of Chemical Engineering at Carnegie Mellon University. He received a BS in Chemistry from the California Institute of Technology and a $\mathrm{PhD}$ in Chemistry from the University of California at Berkeley. His research expertise is in the field of surface science and surface chemistry with emphasis on catalysis, corrosion, chirality and enantioselectivity. In addition, his group has developed tools and methods for high throughput study of alloy surfaces spanning alloy composition space and for studies of structure sensitive properties of crystalline surfaces spanning surface orientation space.

\section{Introduction}

Molecular chirality refers to the geometrical property by which a molecule is non-superimposable on its mirror image. Two enantiomers (the mirror images of a chiral molecule) have identical physical/ chemical properties in achiral environments, rendering them indistinguishable. Chiral molecules are essential to the biochemical processes sustaining life; but common biomolecules such as DNA and proteins are homochiral (present only in one enantiomeric form). Given the biological significance of molecular chirality, understanding enantiospecific interactions of chiral molecules with homochiral environments is of great interest to the agrochemical, pharmaceutical and specialty chemicals industries. While the existence of molecular chirality has been known and studied since being observed by Pasteur in $1848,{ }^{[1]}$ the focus on understanding the interaction of chiral molecules with metal surfaces has emerged only in the last 15-20 years, facilitated by the availability of sophisticated surface sensitive techniques. ${ }^{[2]}$

The study of chiral molecular surface chemistry underpins the development of enantioselective heterogeneous catalysis as an alternative to homogeneously catalyzed synthesis of enantiopure molecules. Avoiding the use of homogeneous chiral organometallic catalysts and the need for their separation from chiral products moti- 
vates development of heterogeneous routes to produce enantiopure chiral chemicals. ${ }^{[3]}$ Chiral separation using chromatography is another field in which the interactions between chiral molecules and surfaces must be understood to design viable chiral stationary phases. ${ }^{[4]}$ In addition to the above, it is hypothesized that the homochirality of life originated from autocatalytic reactions on mineral surfaces in the prebiotic soup. ${ }^{[5]}$ All of these problems motivate our study of chiral surface chemistry.

There are several routes to preparation of chiral surfaces. Surfaces can be rendered chiral by templating chiral molecules onto achiral surfaces. Alternatively, surfaces can be chiral by virtue of their intrinsic atomic structure. ${ }^{[2 b]}$ Such naturally chiral surfaces expose terrace, step and kink structures and, as a result, they lack mirror symmetry. ${ }^{[6]}$ The chirality of the surface may influence the adsorption energies, ${ }^{[7]}$ orientations ${ }^{[8]}$ and aggregation of chiral adsorbates. ${ }^{[7 a, 9]}$

In this paper, we discuss experiments that combine isotopic labelling with surface sensitive techniques to demonstrate both chiral molecular recognition and homochiral aggregation of aspartic acid (Asp, $\mathrm{HOOCCH}\left(\mathrm{NH}_{2}\right) \mathrm{CH}_{2} \mathrm{COOH}$ ) adsorbed on naturally chiral $\mathrm{Cu}(643)^{R \& S}$ surfaces. We provide a qualitative understanding of the Asp decomposition mechanism and a quantitative framework to describe its enantiospecific aggregation on surfaces having different structures. These are prerequisites to developing a predictive understanding of enantiomer surface chemistry.

The most common technique used to probe enantiomer-surface interactions is Scanning Tunneling Microscopy (STM).[10] However, STM suffers from the challenge that the absolute chirality of most adsorbed enantiomers cannot be determined by STM due to insufficient resolution. As a result, the majority of studies of chiral adsorbates on surfaces have used enantiopure molecules rather than enantiomer mixtures, ${ }^{[10,11]}$ obviating the possibility of studying enantiomer aggregation under conditions in which homochiral and heterochiral aggregation compete with one another. An alternative to using STM to study enantiomer mixtures on surfaces is to use mixtures in which one enantiomer is isotopically labelled, thereby allowing discrimination using mass spectrometry. ${ }^{[12]}$ This is viable for chiral molecules (such as amino acids ${ }^{[7 a, 9 b, 13]}$ ) that are readily available in a variety of enantiomerically pure, isotopomeric forms, making them especially attractive for study using temperature programmed reaction (TPR) or desorption (TPD) measurements. In this work, we have used mixtures of unlabeled D-Asp and $4-{ }^{13} \mathrm{C}-\mathrm{L}$-Asp or $1,4-{ }^{13} \mathrm{C}_{2}$-L-Asp adsorbed on the naturally chiral $\mathrm{Cu}(643)^{R \& S}$ surfaces.

Chiral molecular recognition and aggregation on surfaces has been studied in the recent past using adsorbates such as tartaric acid (TA) and Asp. ${ }^{[7 a, 9,13,14]}$ On naturally chiral surfaces such as $\mathrm{Cu}(17,5,1)^{R \& S}, \mathrm{Cu}(651)^{R \& S}$ and $\mathrm{Cu}(531)^{R \& S}$ TA enantiomers decompose enantiospecifically with kinetics that depend on both the structure and chirality of the surfaces. ${ }^{[15]}$ Asp enantiomers in solution have demonstrated enantiospecific surface chemistry by binding enantioselectively to chiral surfaces of calcite crystals. ${ }^{[16]}$ Exposure of the $\mathrm{Cu}(3,1,17)^{R \& S}$ surfaces to a flux of racemic DL-Asp results in preferential adsorption of L-Asp on $\mathrm{Cu}(3,1,17)^{R}$ and D-Asp on $\mathrm{Cu}(3,1,17)^{S}$. ${ }^{[13]}$ Asp has also been shown to decompose enantiospecifically on chiral $\mathrm{Cu}(643)^{R \& S}$ surfaces. ${ }^{[12]}$ Probing homochiral versus heterochiral enantiomer aggregation has been facilitated by the use of enantiospecific isotopic labelling to measure precisely the ratio of $\mathrm{D}$ - to $\mathrm{L}$-enantiomer coverages, $\theta / \theta$, on a surface. ${ }^{[7 a, 9,14 b, c]}$ On the chiral $\mathrm{Cu}(653)^{R \& S}$ surfaces, regardless of surface chirality, adsorption amplifies enantiomeric excess relative to that of the gas phase, $\left|e e_{s}\right|>\left|e e_{g}\right|$, where $e e_{s}=$ $\left(\theta_{\mathrm{D}}-\theta_{\mathrm{L}}\right) /\left(\theta_{\mathrm{D}}+\theta_{\mathrm{L}}\right)$ and $e^{g} e_{g}=\left(P_{\mathrm{D}}-P_{\mathrm{L}}\right) /$ $\left(P_{\mathrm{D}}+P_{\mathrm{L}}^{\mathrm{L}}\right)$. This amplification of $e e^{\mathrm{D}}$ relative to $e e_{g}$ was also observed during adsorption of non-racemic mixtures of Asp on the achiral $\mathrm{Cu}(111)$ surface. ${ }^{[14 c]}$ Collectively, these observations suggest that adsorption of chiral mixtures is governed by a competition between enantiospecific adsorption energetics and the energetics of enantiomer aggregation. ${ }^{99}$ ]

When mixtures of enantiomers crystallize in $3 \mathrm{D}$, it is generally accepted that heterochiral aggregation is overwhelmingly preferred $(\sim 90 \%)$ over homochiral aggregation. ${ }^{[17]}$ However, a recent review of chiral and prochiral adsorbates adsorbed onto various surfaces as racemic mixtures did not find any preference for either homochiral or heterochiral aggregation in 2D layers on surfaces. ${ }^{[14 a]}$ The ability to make such measurements of homochiral vs heterochiral aggregation on model adsorbates such as amino acids can shed light onto the underlying mechanisms of enantiomer aggregation on surfaces.

In the following sections, we demonstrate that through a combination of nonlinear decomposition kinetics and chiral recognition, the rate constants for Asp decomposition on $\mathrm{Cu}(643)^{R \& S}$ surfaces are amplified to yield an enantiospecific difference in decomposition rates of a factor of 3-6. Also, using gas-surface equilibrium adsorption experiments, we show that Asp on $\mathrm{Cu}(643)^{R \& S}$ exhibits weak homochiral aggregation with a free energy of aggregation of $\sim 2.2 \mathrm{~kJ} / \mathrm{mol}$. These findings on enantio- mer aggregation are compared with similar observations of Asp adsorption on $\mathrm{Cu}(111)$, $\mathrm{Cu}(3,1,17)^{R \& S}$ and $\mathrm{Cu}(653)^{R \& S} \cdot{ }^{[9 \mathrm{a}, 14 \mathrm{c}]}$

\section{Experimental Section}

The experiments were performed in an ultra-high vacuum (UHV) chamber operating at a base pressure of $10^{-10}$ Torr. The UHV chamber is equipped with a $(x, y, z$, $\theta)$ manipulator which is used to position the crystal in the chamber, an $\mathrm{Ar}^{+}$sputter ion gun to clean the surface, and low energy electron diffraction (LEED) optics to verify the surface structure. Asp, in powder form, was sublimated onto the $\mathrm{Cu}(643)^{R \& S}$ surfaces using a Kentax ${ }^{\circledR}$ two-cell organic evaporator. Finally, an Extrel ${ }^{\circledR}$ quadrupole mass spectrometer was used to detect gas phase species being introduced into the chamber and species desorbing from the surface.

The naturally chiral $\mathrm{Cu}(643)^{R \& S}$ crystal (Monocrystals company, Ohio) is $10 \mathrm{~mm}$ in diameter by $2 \mathrm{~mm}$ thick and is spot-welded to Ta wires at the end of the manipulator. The $\mathrm{Cu}(643)$ surface structure consists of (111) terraces, with (100) step facets and (110) kinks (Fig. 1). ${ }^{[6 c]}$ The step and kink facets together form $(310)^{R}$ or $(310)^{S}$ steps. The single crystal disk exposes the $\mathrm{Cu}(643)^{R}$ surface on one side and its enantiomer, $\mathrm{Cu}(643)^{S}$, on the opposite side. A K-type (chromel-alumel) thermocouple was spot-welded onto the edge of the crystal for temperature measurement. Using liquid nitrogen, the crystal was cooled to $90 \mathrm{~K}$ and it was heated resistively to 1000 $\mathrm{K}$. The crystal was cleaned using multiple $\mathrm{Ar}^{+}$sputter/anneal cycles consisting of sputtering with $P_{4 x}=5 \times 10^{-5}$ Torr and an $\mathrm{Ar}^{+}$energy of $1 \mathrm{keV}$. During sputtering, the crystal was held at $850 \mathrm{~K}$ followed by annealing at $900 \mathrm{~K}$. In addition to using electron backscatter diffraction to verify the crystal surface orientation, LEED was used after sputter/anneal cycles to verify the long range order and chirality of the surface.

Unlabelled D-Asp (Sigma Aldrich, 99 atom\%), $4-{ }^{13} \mathrm{C}-\mathrm{L}-\mathrm{Asp}$ (Cambridge Isotope Laboratories, 99 atom\%, $\mathrm{HOOCCH}\left(\mathrm{NH}_{2}\right)$ $\left.\mathrm{CH}_{2}{ }^{13} \mathrm{COOH}\right)$ and $1,4-{ }^{13} \mathrm{C}_{2}$-L-Asp (Cambridge Isotope Laboratories, 99 atom $\left.\%, \quad \mathrm{HOO}^{13} \mathrm{CCH}\left(\mathrm{NH}_{2}\right) \mathrm{CH}_{2} \mathrm{C}^{13} \mathrm{OOH}\right)$ were used for the experiments. The $\mathrm{D}-$ and L-Asp were loaded into two resistively heated quartz crucibles in the Kentax evaporator. The vial temperatures were controlled independently at 400-420 K to achieve sublimation of Asp vapor with independently controlled fluxes onto the crystal surface. The vials were positioned $3-5 \mathrm{~cm}$ from the crystal to achieve uniform coverage, $\theta$, across the surface. During heating, Asp/Cu(643) ${ }^{R \& S}$ decomposes into $\mathrm{CO}_{2}, \mathrm{H}_{2}$ and $\mathrm{N} \equiv \mathrm{CCH}_{3}$, all of which desorb rapidly into the gas phase. $\mathrm{CO}_{2}$ from unla- 
Fig. 1. Ideal $\mathrm{Cu}(643)^{\text {Res }}$ structures showing terraces, steps and kinks characteristic of high Miller index surfaces. The (643) surface consists of (111) terraces separated by monoatomic steps formed of (100) close-packed runs with (110) kinks. The sense of rotation between (111), (100) and (110) microfacets renders the structure chiral.

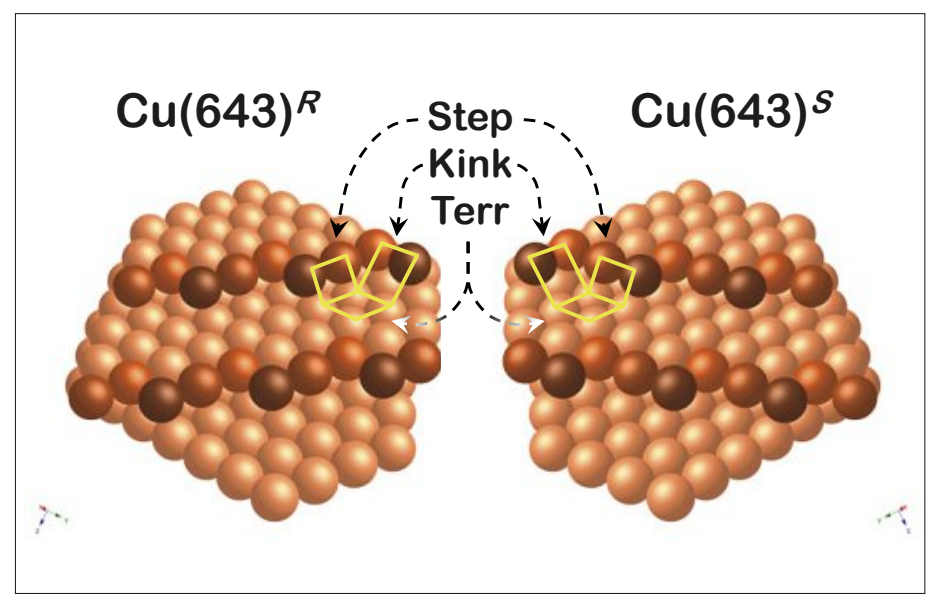

monolayers/min. After exposure of the surface for a controlled period of time, the D-/L-Asp coverage ratio, $\theta_{\mathrm{D}} / \theta_{\mathrm{L}}$, was determined by TPRS from the areas under the $m / z=44$ and 45 peaks. First, it was necessary to determine the D-/L-Asp flux ratio in the gas phase, $F_{\mathrm{D}} / F_{\mathrm{L}}$, at given vial temperatures. For this, the vial temperatures needed to deliver a fixed $F_{\mathrm{D}} / F_{\mathrm{L}}$ to the clean surface were determined by depositing for short time periods to yield low Asp coverages. At low total coverages, $\theta<<1$, the coverage ratio, $\theta_{\mathrm{D}} / \theta_{\mathrm{L}}$, is a measure of the relative fluxes, $F_{\mathrm{D}} / F_{\mathrm{L}}$, of D- and $\mathrm{L}$-Asp from the gas phase. For exposures greater than that necessary to reach saturation coverage, the value of $\theta_{D} / \theta_{L}$ may differ from $F_{D} /$ $F$ due to enantiospecific adsorption and/ or enantiospecific aggregation, as demonstrated below.

\section{Results}

\subsection{Mechanism and Kinetics of Asp Decomposition on $\mathrm{Cu}(643)$}

\subsubsection{Decomposition Mechanism of Asp/Cu(643)}

The adsorption and decomposition of Asp has been studied previously on the achiral $\mathrm{Cu}(110)$ surface. ${ }^{[12]}$ Using X-ray photoelectron spectroscopy (XPS), it was found that Asp is present in its zwitterionic form in the multilayer $\left({ }_{2}^{-} \mathrm{CCH}\left(\mathrm{NH}_{3}{ }^{+}\right)\right.$ $\mathrm{CH}_{2} \mathrm{COOH}$ ), as also found in its solid form. However, Asp adsorbed on $\mathrm{Cu}(110)$

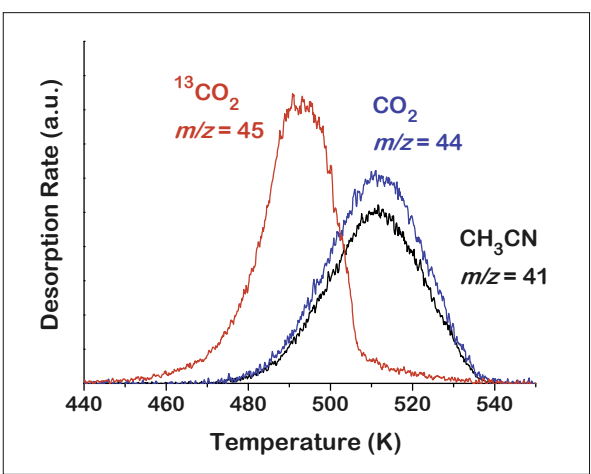

Fig. 2. TPRS of $4-{ }^{13} \mathrm{C}-\mathrm{L}-\mathrm{Asp}$ decomposition on $\mathrm{Cu}(643)^{R}$. Isotopically labelling the $\mathrm{C} 4$ carbon of Asp allows resolution of the ${ }^{13} \mathrm{CO}_{2}$ and $\mathrm{CO}_{2}$ isotopomers formed sequentially during step 1 (C3-C4 cleavage) and step 2 (C1-C2 cleavage) of Asp decomposition. The remaining $\mathrm{C}_{2} \mathrm{NH}_{5}$ fragment yields $\mathrm{N} \equiv \mathrm{CCH}_{3} . \theta_{\mathrm{L}}=0.5$ and heating rate $=2 \mathrm{~K} / \mathrm{s}$.

in the monolayer is doubly deprotonated to form anionic biaspartate $\left({ }^{-} \mathrm{O}_{2} \mathrm{CCH}\left(\mathrm{NH}_{2}\right)\right.$ $\mathrm{CH}_{2} \mathrm{COO}^{-}$) with the carboxylate groups presumably coordinated to $\mathrm{Cu}$ atoms. We assume that the same is true on the $\mathrm{Cu}(643)^{R \& S}$ surfaces.

To determine the decomposition mechanism of Asp on $\mathrm{Cu}(643)^{R \& S}$ during heating, several different isotopomers of Asp were adsorbed on the surface and the gas phase decomposition products were identified using the mass spectrometer. A typical TPR spectrum obtained using $4-{ }^{13} \mathrm{C}-\mathrm{L}$-Asp on $\mathrm{Cu}(643)^{R}\left(\theta_{\mathrm{L}}=0.5\right.$, heating rate $\left.2 \mathrm{~K} / \mathrm{s}\right)$ is shown in Fig. 2. The signal at $m / z=45$ with a peak temperature of $T_{p}=485 \mathrm{~K}$ arises from ${ }^{13} \mathrm{CO}_{2}$ generated by cleavage of the C3-C4 bond. The signal at $m / z=44\left(T_{p}=\right.$ $512 \mathrm{~K}$ ) arises from $\mathrm{CO}_{2}$ generated by cleavage of the $\mathrm{C} 1-\mathrm{C} 2$ bond. In addition to the $\mathrm{CO}_{2}$ peaks, a peak at $\mathrm{m} / \mathrm{z}=41$ with $T_{p}=$ $512 \mathrm{~K}$ arises from desorption of acetonitrile $\left(\mathrm{N} \equiv \mathrm{CCH}_{3}\right.$ ) generated from the $\mathrm{C}_{2} \mathrm{NH}_{5}$ fragment remaining after decarboxylation of the adsorbed Asp. The same products are observed in a similar sequence on $\mathrm{Cu}(110)^{[12]}$ and in ongoing work on $\mathrm{Cu}(100)$.

The three-step decomposition pathway of doubly deprotonated Asp adsorbed on $\mathrm{Cu}(643)^{R}$ as deduced from the TPRS of different Asp isotopomers is summarized below. of the opposite enantiomer for different exposure times while the crystal temperature was held constant at $400 \mathrm{~K}$. The flux was sufficient to deposit a monolayer of Asp in 10-15 min. After each exposure, TPRS was conducted and the coverages of $\mathrm{D}$-Asp and $1,4-{ }^{13} \mathrm{C}_{2}$-L-Asp were estimated from the areas under the $m / z=44$ and 45 peaks.

The equilibrium adsorption experiments were performed with the $\mathrm{Cu}(643)^{R \& S}$ surface held at $400 \mathrm{~K}$ and sublimating both D- and $1,4-{ }^{13} \mathrm{C}_{2}-\mathrm{L}-\mathrm{Asp}$ from two separate vials at constant, independently controlled fluxes, typically ranging from $0.05-0.15$

adsorption step 1

step 2 step 3 
Asp adsorbs onto the $\mathrm{Cu}$ surface by chemisorption in the biaspartate form, losing the two carboxylate hydrogen atoms in the process. The biaspartate species then undergoes a three-step decomposition wherein the $\mathrm{C} 4$ carboxylate group, followed by the $\mathrm{C} 1$ carboxylate desorb as $\mathrm{CO}_{2}$, leaving behind a surface intermediate $\mathrm{C}_{2} \mathrm{NH}_{5}$ which immediately decomposes to yield acetonitrile and $\mathrm{H}_{2}$ desorption.

\subsubsection{Surface Explosion Kinetics of Asp/Cu(643)}

To study the kinetics of the $4-{ }^{13} \mathrm{C}_{2}-\mathrm{L}-$ Asp decomposition reaction on $\mathrm{Cu}(643)^{S}$, TPRS experiments were performed starting at different initial values of $\theta$. Fig. 3 , left panel shows the sum of the desorption signals for $\mathrm{CO}_{2}$ and ${ }^{13} \mathrm{CO}_{2}$ versus temperature. As the initial value of $\theta$ increased, the peak temperatures increased and the peak widths decreased. Such behavior is attributed to a vacancymediated surface explosion mechanism in which the decomposition step requires the presence of a vacancy adjacent to the decomposing adsorbate; hence, the rate law depends on both the adsorbate and vacancy coverages, $r=\theta(1-\theta) .{ }^{[18]}$ This type of decomposition kinetics has also been observed for TA decomposition on $\mathrm{Cu}$ surfaces. ${ }^{[15,19]}$ At low adsorbate coverages, the rate is effectively first-order in adsorbate coverage because $(1-\theta) \approx 1$. As the initial coverage approaches $\theta=1$ (saturation coverage), (1- $\theta)$ goes to zero leading to higher peak decomposition temperatures. When the initial coverage is very close to saturation, the autocatalytic increase in the vacancy coverage during onset of the decomposition reaction leads to a non-linear increase in reaction rate and consequently, a very narrow peak width. An extreme manifestation of a surface explosion reaction is seen in the TPRS of TA/ $\mathrm{Cu}(110)$ where peak widths $<1 \mathrm{~K}$ are observed. ${ }^{[15]}$ Such narrow peak widths were achievable with TA because, unlike Asp, all products of TA decomposition appear in the gas phase simultaneously, presumably rate-limited by the initial $\mathrm{C}-\mathrm{C}$ bond cleavage to yield $\mathrm{CO}_{2}$.

\subsubsection{Chiral Recognition/Enantiospe- cific Decomposition of Asp/Cu(643) ${ }^{R \& S}$}

Using ${ }^{4-13} \mathrm{C}-\mathrm{L}-\mathrm{Asp}$ it is possible to resolve the kinetics of the two decarboxylation steps in the Asp decomposition mechanism on $\mathrm{Cu}(643)^{R \& S}$, allowing independent determination of the peak $\mathrm{CO}_{2}$ desorption temperatures. The right-hand panel of Fig. 3 plots the two peak $\mathrm{CO}_{2}$ desorption temperatures arising from $4-{ }^{13} \mathrm{C}-\mathrm{L}$-Asp decomposition as functions of the initial coverage, $\theta$, on both $\mathrm{Cu}(643)^{R \& S}$. The blue and red squares represent step $1(\mathrm{C} 3-\mathrm{C} 4$ cleavage) peak temperatures at different $\theta_{L}$
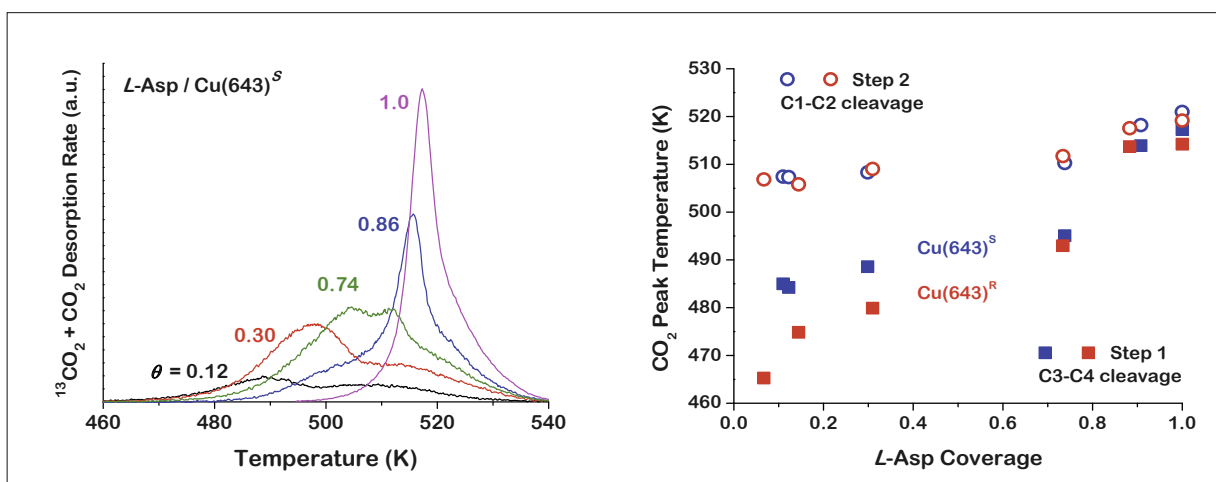

Fig. 3. Left panel: TPRS of L-Asp/Cu(643) $)^{R}$ at different initial coverages, $\theta_{L}$. As $\theta_{\mathrm{L}}$ increases from 0.12 to 0.74 , two distinct $\mathrm{CO}_{2}$ desorption peaks can be seen arising from sequential cleavage of the C3-C4 and C1-C2 bonds. As $\theta_{\mathrm{L}}$ tends towards 1, the peak temp increases from $490 \mathrm{~K}$ to $520 \mathrm{~K}$ and the peak width decreases from $\sim 40 \mathrm{~K}$ to $\sim 8 \mathrm{~K}$. These characteristics suggest that the decomposition of Asp proceeds via an autocatalytic, vacancy-mediated explosion mechanism. Right panel: Peak temperature versus initial $\theta$ for step 1 (squares, C3-C4 cleavage) and step 2 (circles, C1-C2 cleavage) during $4-{ }^{13} \mathrm{C}-\mathrm{L}-\mathrm{Asp} / \mathrm{Cu}(643)^{R \& S}$ decomposition. The red symbols represent $4-{ }^{13} \mathrm{C}-\mathrm{L}-\mathrm{Asp} / \mathrm{Cu}(643)^{R}$ while the blue symbols represent $4-{ }^{13} \mathrm{C}-\mathrm{L}-\mathrm{Asp} / \mathrm{Cu}(643)^{S}$. For $\theta=$ $0.1-0.4$, there is an enantiospecific peak temperature difference of $\sim 10 \mathrm{~K}$ for step 1 but no detectable enantiospecificity for step 2 .

on $\mathrm{Cu}(643)^{R}$ and $\mathrm{Cu}(643)^{S}$, respectively. The blue and red circles represent step 2 (C1-C2 cleavage) peak temperatures. There is a significant difference between the peak temperatures of step 1 and step 2 decomposition, especially at low $\theta$. More importantly, on the $\mathrm{Cu}(643)^{R}$ and $\mathrm{Cu}^{L}(643)^{S}$ surfaces there is $>10 \mathrm{~K}$ enantiospecific difference in the peak temperatures for step 1 . This difference gradually reduces as $\theta_{L}$ approaches 1. Thus, the Asp decomposition kinetics on the naturally chiral $\mathrm{Cu}(643)^{R \& S}$ surfaces reveal chiral recognition and enantiospecificity. In contrast to step 1 , there is no enantiospecific difference in the peak temperatures for step 2 at any value of $\theta$. This is quite surprising given that step 1 , cleavage of the $\mathrm{C} 3-\mathrm{C} 4$ bond, does not involve the chiral center at $\mathrm{C} 2$ whereas step 2 does.

\subsubsection{Amplification of Enantiospecific Decomposition Rates of Asp/Cu(643) R\&S}

Fig. 4 shows the TPRS of D-Asp and $4-{ }^{13} \mathrm{C}-\mathrm{L}-\mathrm{Asp}$ (red and blue lines, respectively) on $\mathrm{Cu}(643)^{R}$ at $\theta=1$. As discussed elsewhere, there is a statisically significant difference of $\sim 3 \mathrm{~K}$ in the L-Asp and $\mathrm{D}$ Asp peak decomposition temperatures on $\mathrm{Cu}(643)^{R}$, with L-Asp decomposition occurring at lower temperature than D-Asp. ${ }^{[12]}$ The opposite enantiospecificity is observed for $\mathrm{Asp} / \mathrm{Cu}(643)^{S}$. Note that, consistent with the data in Fig. 3 showing enantiospecificity of step 1 , the peak desorption temperatures at $\theta=1$ are dominated by the first step in the Asp decomposition mechanism. The TPRS in Fig. 4 corroborate our finding from Fig. 3 that the naturally chiral $\mathrm{Cu}(643)^{R \& S}$ surfaces decompose Asp with enantiospecific kinetics. $\mathrm{Cu}(643)^{R}$ decomposes L-Asp faster than D-Asp while
$\mathrm{Cu}(643)^{S}$ decomposes D-Asp faster than L-Asp, demonstrating diastereomerism. ${ }^{[12]}$ Fig. 4 also shows the ratio of the rates of L- and D-Asp decomposition on $\mathrm{Cu}(643)^{R}$. The initial decomposition rates at high coverage exhibit enantiospecificity in the range $r_{\mathrm{L}} / r_{\mathrm{D}}=3$ to 6 . For a first-order process such as desorption, a $3 \mathrm{~K}$ temperature difference in the peak rates observed in a TPR or TPD experiment would correspond to a difference in reaction energetics of $\sim 1$ $\mathrm{kJ} / \mathrm{mol}$ and yield a difference of $\sim 1.3$ in reaction rates at $500 \mathrm{~K} .{ }^{[7 \mathrm{~b}, 20]}$ However, the highly non-linear kinetics of the surface explosion mechanism amplify the enantiospecific difference on chiral surfaces to $>3$. The same amplification is observed for D-/L-Asp decomposition on $\mathrm{Cu}(643)^{S}$ but favoring D-Asp over L-Asp.

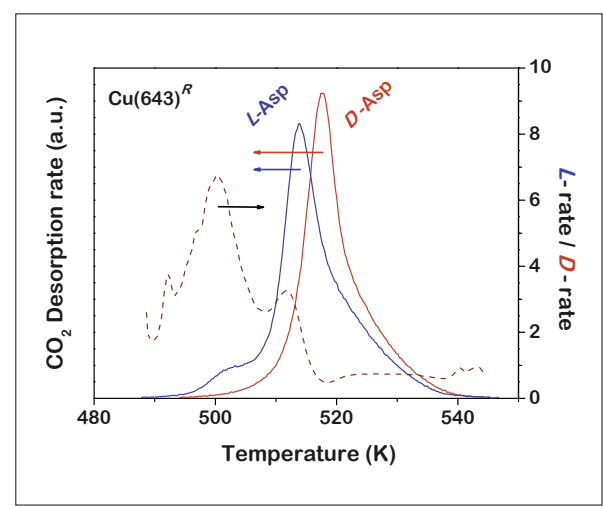

Fig. 4. TPRS of L-Asp and D-Asp (in blue and red, respectively) on $\mathrm{Cu}(643)^{R}$ at $\theta=1$ and the ratio of the decomposition rates (dashed curve). The combination of non-linear decomposition kinetics and the chiral surface amplifies small difference of $\sim 3 \mathrm{~K}$ in peak temperature into a factor of 3-6 difference in enantiospecific decomposition rates. 


\subsection{Competitive Equilibrium Ad- sorption of D-/L-Asp Mixtures on $\mathrm{Cu}(643)^{R \& S}$}

In addition to the enantiospecific decomposition kinetics of D- and L-Asp on $\mathrm{Cu}(643)^{R \& S}$ revealed in the previous section, D- and L-Asp should exhibit enantiospecific adsorption energies on the $\mathrm{Cu}(643)^{R \& S}$ surfaces. These should be reflected in the relative coverages of the two enantiomers adsorbed in equilibrium with an enantiomer mixture in the gas phase. In order to establish equilibrium between a mixture of enantiomers in the gas phase and a mixture adsorbed on a surface, there must be an exchange process by which gas phase enantiomers can displace adsorbed enantiomers. To verify this for $\mathrm{Asp} / \mathrm{Cu}(643)^{R \& S}$, displacement experiments were conducted by starting with either D-Asp or $1,4-{ }^{13} \mathrm{C}_{2}-\mathrm{L}$ Asp at $\theta=1$ on the $\mathrm{Cu}(643)^{R}$ or $\mathrm{Cu}(643)^{S}$ surfaces and then exposing them to a flux of the opposite enantiomer for various periods of time, with the crystal held at $T=$ $400 \mathrm{~K}$. After each exposure, TPRS was performed while monitoring the signals at $\mathrm{m} / \mathrm{z}=44$ and 45 to determine the relative amounts of $\mathrm{D}^{-}$and $1,4-{ }^{13} \mathrm{C}_{2}$-L-Asp on the surface.

On a $\mathrm{Cu}(643)^{R \& S}$ surface initially covered with L-Asp, increasing exposure to D-Asp should decrease $\theta$ and increase $\theta$ monotonically until all of the L-Asp has been exchanged for D-Asp. Fig. 5 left panel plots $\theta$ and $\theta$ as functions of exposure time for $\mathrm{Cu}(643)^{S}$ initially saturated with either D-Asp (square symbols and dotted lines) or L-Asp (circles and dashed lines) and then exposed to a flux of the opposite enantiomer. The data reveal the continuous displacement of the initial adsorbate and accumulation of the species from the gas phase. On $\mathrm{Cu}(643)^{S}$ at $400 \mathrm{~K}$, D-Asp clearly displaces L-Asp faster than L-Asp displaces D-Asp. The right panel of Fig. 5 shows the same data but obtained on $\mathrm{Cu}(643)^{R}$. $\mathrm{On} \mathrm{Cu}(643)^{R}$ at $400 \mathrm{~K}$, D-Asp displaces L-Asp (round symbols and dashed lines) more slowly than L-Asp displaces D-Asp (square symbols and dotted lines). These data demonstrate rapid self-displacement of Asp enantiomers, enantiospecificity with respect to the surface chirality and diastereomerism.

For each of the four data sets shown in Fig. 5, the coverages of the displacing and the initially adsorbed enantiomers were fit to exponential growth and decay equations, $\theta_{\text {disp }}=\left(1-e^{-k t}\right)$ and $\theta_{\text {init }}=e^{-k t}$ to determine the displacement rate constant, $k$. The rate constant for $\mathrm{D}$-Asp displacing L-Asp on $\mathrm{Cu}(643)^{R}$ is $k_{R}^{D / L}=0.012 \mathrm{~min}^{-1}$ \pm 0.0002 and that for L-Asp displacing $\mathrm{D}^{-}$ Asp on $\mathrm{Cu}(643)^{R}$ is $k_{R}^{L / D}=0.025 \mathrm{~min}^{-1} \pm$ 0.003 . These demonstrate enantiospecificity in that they differ from one another. Similarly, on $\mathrm{Cu}(643)^{S}$ the rate constant
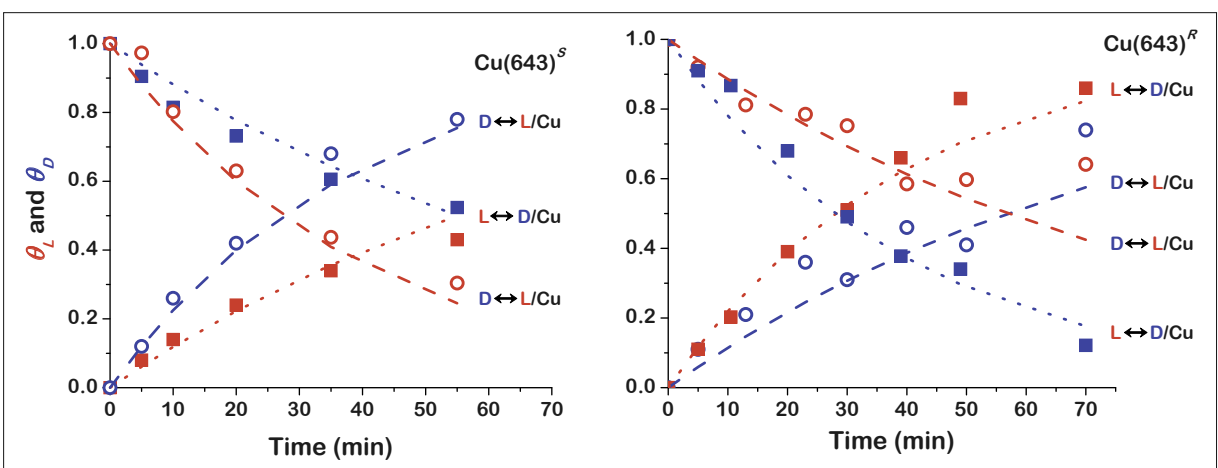

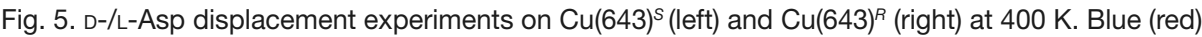
symbols and lines represent $\theta_{D}\left(\theta_{L}\right)$. D-Asp displacement of L-Asp is shown with dashed lines; L-Asp displacement of D-Asp with dotted lines. Left: On Cu(643)S D-Asp displaces L-Asp faster than L-Asp displaces D-Asp. Right: The opposite occurs on $\mathrm{Cu}(643)^{R}$. The displacement rates, fitted to a simple exponential decay and growth equations (dashed and dotted lines), are found to be enantiospecific. The rate constant for L-Asp displacement of D-Asp on $\mathrm{Cu}(643)^{R}\left(k_{R}^{D / L}=0.025 \mathrm{~min}^{-1}\right)$ differs from that on $\mathrm{Cu}(643)^{S}\left(k_{S}^{L / D}=0.013 \mathrm{~min}^{-1}\right)$. Diastereomerism is demonstrated by the fact that $k_{S}^{L / D} \cong k_{R}^{D / L}=0.012 \mathrm{~min}^{-1}$ and $k_{S}^{D / L} \cong k_{R}^{L / D}=0.026 \mathrm{~min}^{-1}$. Total incident flux $F_{\text {Asp }} \cong 0.1 \mathrm{ML} / \mathrm{min}$.

for $\mathrm{L}$-Asp displacing $\mathrm{D}$-Asp is $k_{S}^{L / D}=$ $0.013 \mathrm{~min}^{-1} \pm 0.002$ and that for $\mathrm{D}$-Asp displacing L-Asp is $k_{S}^{D / L}=0.026 \mathrm{~min}^{-1} \pm$ 0.003 . While these rate constants reveal enantiospecificity of the displacement kinetics, they also reveal diastereomerism, $k_{S}^{L / D}=k_{R}^{D / L} \neq k_{S}^{D / L}=k_{R}^{L / D}$

It is worth noting that the displacement process is extremely efficient. The displacement experiments were conducted with incident fluxes of $F_{A c p} \cong 0.1 \mathrm{ML} / \mathrm{min}$. If every incident Asp displaced an adsorbed Asp then the time constant for replacement of $50 \%$ of the initially adsorbed enantiomer would be $\sim 10 \mathrm{~min}$. What we measure are time constants of $\sim 40$ or 80 min depending upon the combination of Asp enantiomers and the chirality of the surface. Little is known about the details of these displacement reactions but the process must involve the transfer of two $\mathrm{H}$-atoms between the carboxylate groups of the incident Asp and the displaced Asp. The efficiency with which this occurs indicates that the barrier to this proton exchange process is very low.

\subsection{Equilibrium Adsorption of D-/L- Asp on $\mathrm{Cu}(643)^{R \& S}$}

In order to determine whether D-/L-Asp enantiomers adsorb enantiospecifically on the $\mathrm{Cu}(643)^{R \& S}$ surfaces, equilibrium adsorption experiments were performed by exposing the surfaces held at $T=400 \mathrm{~K}$ to gas phase mixtures of $\mathrm{D}$-/L-Asp with various values of enantiomeric ee $\left.=\left(\mathrm{P}_{\mathrm{D}}-\mathrm{P}_{\mathrm{L}}\right) / \mathrm{P}_{\mathrm{D}}+\mathrm{P}_{\mathrm{L}}\right)$ Our demonstration that ${ }^{g}$ the pure enantiomers displace one another on the $\mathrm{Cu}(643)^{R \& S}$ surfaces indicates that exposing the surfaces to a constant flux of a D-/L-Asp mixture should establish equilibrium between the gas and adsorbed phases. ${ }^{[13]}$ The enantiomeric excess on the surface, $e e_{g}$, will not necessarily be the same as that in the gas phase. If one enantiomer has a higher heat of adsorption than the other, then its fractional coverage on the surface will be higher than its fractional partial pressure in the gas phase. Alternatively, if there is a tendency for adsorbed enantiomers to aggregate homochirally, the surface will become enriched in the majority gas phase enantiomer, and $e e$ will be amplified relative to $e e_{g}{ }^{[14 \mathrm{c}]}$ Similarly, if the enantiomers tend to aggregate heterochirally, then $e e$, will be attenuated relative to $e e_{g} .{ }^{[7 \mathrm{a}]}$

The equilibrium adsorption of D-Asp and $1,4-{ }^{13} \mathrm{C}_{2}$-L-Asp was studied on both $\mathrm{Cu}(643)^{R \& S}$ surfaces. In these experiments, the $\mathrm{Cu}(643)$ crystal was held at $400 \mathrm{~K}$ and either the $R$ or $S$ surface was exposed to a gas phase flux of D-/L-Asp for a predetermined period of time. At that point, the shutter was closed, the surface temperature was quenched, the surface was moved in front of the mass spectrometer and TPRS was conducted while monitoring signals at $\mathrm{m} / \mathrm{z}=44$ and 45 to determine the relative coverages of D- and $1,4-{ }^{13} \mathrm{C}_{2}$-L-Asp on the surface. Fig. 6 shows $\theta / \theta^{2}$ as a function of exposure time using six different ratios of enantiomer partial pressures in the gas phase, $P_{\mathrm{D}} / P_{\mathrm{L}}$. The fluxes of the two Asp enantiomers were controlled such that the total was $\sim 0.1 \mathrm{ML} / \mathrm{min}$. When $P_{\mathrm{D}} / P_{\mathrm{L}}=1$ (blue squares), the enantiomer coverage ratio remains $\theta_{\mathrm{D}} / \theta_{\mathrm{L}}=1$ for exposures up to $60 \mathrm{~min}$. This clearly indicates that there is no detectable enantiomeric preference for Asp adsorption on $\mathrm{Cu}(643)^{R \& S}$. When exposed to a gas phase with $P_{\mathrm{D}} / P_{\mathrm{L}}=1.3$ (black filled circles), the value of $\theta_{\mathrm{D}} / \theta_{\mathrm{L}}$ is $\sim 1.3$ initially, but once the coverage saturates, $\theta_{\mathrm{D}} / \theta_{\mathrm{L}}$ starts to increase with exposure and approaches a steady-state at $\theta_{\mathrm{D}} / \theta_{\mathrm{L}}=2$. Similarly, when the surface is exposed to a gas phase mixture with $P_{\mathrm{D}} / P_{\mathrm{L}}=0.43$ (green filled circles), the value of $\theta_{\mathrm{D}} / \theta_{\mathrm{L}}$ decreases after reaching a monolayer coverage and reaches steady-state at $\theta_{\mathrm{D}} / \theta_{\mathrm{L}}=0.33$. On ex- 
posure of the $\mathrm{Cu}(643)^{S}$ surface to $P_{\mathrm{D}} / P_{\mathrm{L}}=$ 0.28 (cyan filled circles), the value of $\theta_{\mathrm{D}}^{\mathrm{L}} / \theta_{\mathrm{L}}$ equilibrates at $\sim 0.23$.

In order to demonstrate that the value of $\theta / \theta_{\mathrm{L}}$ reached at long exposure times is indeed an equilibrium value, experiments were performed by exposing the surfaces to a given $P / P$ until the coverage saturates at $\theta=1$ and $\theta / \theta_{\mathrm{L}}=P / P$. Then the surface was exposed to a D-/L-Asp flux with $P_{\mathrm{D}} /$ $P$ at a different but previously used value. The goal is to determine whether the final value of $\theta / \theta$ is independent of the initial coverage ratio on the surface; i.e. one can approach the same equilibrium coverage ratio at a given $P / P$ starting with initial coverage ratios that are greater than and less than the equilibrium coverage ratio. Two such sets of experiments are shown in Fig. 6. Starting with $P / P=3.5$ (black open circles), a saturated monolayer with $\theta_{\mathrm{D}} / \theta_{\mathrm{L}}=$ 3.5 is formed. Once the saturated monolayer was formed, the surface was exposed to $P_{1} / P_{\mathrm{L}}=1.3$ for various exposure times. The value of $\theta / \theta$ then drops and equilibrates at $\theta / \theta=1.9$, the same as the value reached when initially exposing a clean surface to a flux with $P_{\mathrm{D}} / P_{1}=1.3$ (black filled circles). In another such experiment, starting with $\theta / \theta_{L}=0.25$ (purple open circles) and subsequently exposing the surface to $P / P=$ 1.1, the surface equilibrates at $\theta / \theta=1.35$, the same as the value reached when exposing the initially clean surface to $P / P=1.1$ (purple filled circles). These experiments demonstrate that the steady-state value of $\theta_{\mathrm{D}} / \theta_{\mathrm{L}}$ measured by this method depends only on the $P / P$ ratio in the gas phase and not the initial enantiomer coverage ratio on the surface. Thus, the steady-state value of $\theta / \theta$ is a good estimate of the equilibrium coverage ratio.

The equilibrium values of $\theta_{\mathrm{D}} / \theta_{\mathrm{L}}$ measured for various values of $P / P$ can be plotted in the form of an adsorption isotherm relating the equilibrium value of enantiomeric excess on the surface, $e e_{s}=\left(\theta_{\mathrm{D}}\right.$ $\left.-\theta_{\mathrm{L}}\right) /\left(\theta_{\mathrm{D}}+\theta_{\mathrm{L}}\right)$, to that in the gas phase, $e e_{\mathrm{g}}=$ $\left(P_{\mathrm{D}}-P_{\mathrm{L}}\right) /\left(P_{\mathrm{D}}+P_{\mathrm{L}}\right)$. Values of $e e=+1$ or $^{g}-1$ signify enantiopure $\mathrm{D}$ - and L-Asp, respectively. The resulting isotherm measured on the $\mathrm{Cu}(643)^{R \& S}$ surfaces is shown in Fig. 7 (blue squares). The observation that the enantiomer coverage ratio on the $\mathrm{Cu}(643)^{R \& s}$ surfaces remains racemic, $e e_{s}=1$, when exposed to a racemic gas phase flux, $e e_{g}=$ 1 , demonstrates that the surfaces do not a dsorb Asp enantiospecifically. Data points obtained on both the $\mathrm{Cu}(643)^{R \& S}$ surfaces are included in the plot, with both surfaces behaving identically due to absence of any enantiospecificity. Nonetheless, the data points do deviate from the $e e_{s}=e e_{g}$ line. Regardless of surface chirality, adsorption results in local amplification of the majority enantiomer present in the gas phase, $\left|e e_{s}\right|>\left|e e_{g}\right|$. In the absence of enantio- specificity, this auto-amplification is only possible if the adsorbates aggregate homochirally on the surface. ${ }^{[14 a]}$

\section{Discussion}

The adsorption isotherms in Fig. 7 can be modeled to determine the equilibrium constant for homochiral aggregation, as discussed previously.[9a,14c] Briefly, the Langmuir adsorption model (which conventionally does not account for adsorbate-adsorbate interactions) can be modified to account for clustering of adsorbates into aggregates. This clustering of adsorbates can be modelled by fitting the isotherm to two parameters; a cluster size, $n$, describing the number of adsorbate molecules per cluster and an equilibrium constant, $K_{c}$ (homochiral aggregation equilibrium constant). In this case, the adsorbates cluster homochirally into conglomerate clusters rather than racemate clusters. The equilibrium coverages of adsorbate monomers can be written as $\theta_{\mathrm{D}}=K_{a} P \delta$ and $\theta=$ $K P \delta$ where $K_{a}$ is the non-enantiospecific adsorption equilibrium constant and $\delta$ is the fractional coverage of vacancies on the surface. The coverages of D- and L-Asp clusters of $n$ molecules are determined by equilibrium equations, $\theta_{D n}=K_{c} \theta_{D}^{n}$ and $\theta_{L n}=K_{C} \theta_{L}^{n}$. The coverage of the vacancies on the surface is defined by Eqn. (1) below. The molecular enantiomeric excess on the surface, $e e_{s}$, can be defined in terms of the monomer and cluster coverages (Eqn. (2)). Substituting the expression above for $\theta$, $\theta_{\mathrm{L}}, \theta_{\mathrm{Dn}}$ and $\theta_{\mathrm{un}}$ into Eqns (1) and (2) yields Eqns (3) and (4).

$$
\begin{aligned}
& \delta=1-\theta_{D}-\theta_{L}-n \theta_{D n}-n \theta_{L n} \\
& e e_{S}=\frac{\left(\theta_{D}+n \theta_{D n}\right)-\left(\theta_{L}+n \theta_{L n}\right)}{\left(\theta_{D}+n \theta_{D n}\right)+\left(\theta_{L}+n \theta_{L n}\right)} \\
& 0=1-\left(1+K_{a} P\right) \delta-n K_{c}\left(\frac{K_{a} P}{2}\right)^{n}\left(\left(1+e e_{g}\right)^{n}+\left(1-e e_{g}\right)^{n}\right) \delta^{n} \\
& e e_{s}(1-\delta)=K_{a} P\left(e e_{g}\right) \delta+n K_{c}\left(\frac{K_{a} P}{2}\right)^{n}\left(\left(1+e e_{g}\right)^{n}+\left(1-e e_{g}\right)^{n}\right) \delta^{n}
\end{aligned}
$$

Fig. 6. Equilibrium adsorption of $\mathrm{D} / \mathrm{L}-\mathrm{Asp}$ on $\mathrm{Cu}(643)^{R}$ and $\mathrm{Cu}(643)^{S}$ at $400 \mathrm{~K}$ showing $\theta_{\mathrm{D}} / \theta_{\mathrm{L}}$ as a function of time exposed to a flux $\approx 0.1$ $\mathrm{ML} / \mathrm{min}$ ) with a constant $P_{\mathrm{D}} / P_{1}$. The data shown with solid circles was obtained starting with initially clean surfaces. The data shown with open circles was obtained by starting with $\left(\theta_{\mathrm{D}} / \theta_{\mathrm{L}}\right)_{0}=0.25$ (purple) or 3.5 (black) and exposing to $P / P=1.1$ and 1.3 , respectively. Regardless of $\left(\theta_{D} / \theta_{L}\right)_{0}$, the values of $\theta_{D} / \theta_{L}$ approached in steady-state are roughly independent of the initial coverage ratios.
For given values of the parameters $K P$, $K$ and $n$, Eqns (3) and (4) can be solved to yield $e e_{s}$ for any value of $e e_{g}$. Thus, Eqns (3) and (4) can be fit to the experimental data in Fig. 7 to yield estimates of $K P, K$ and $n$. Adsorption under the conditions of the experiment yields saturated monolayer coverages with $\delta \approx 0$. Hence, we can set $K_{a} P>>1$ to yield $\delta \approx 0$, rendering the fit insensitive to the value of $K P$. This leaves just two parameters, $K_{c}$ and $n$, to be determined from the data.

Fig. 7 shows the best fit adsorption isotherms (solid lines) for $\mathrm{Asp} / \mathrm{Cu}(111)$, $\mathrm{Asp} / \mathrm{Cu}(653)^{R \& S}$ and $\mathrm{Asp} / \mathrm{Cu}(643)^{R \& S} \cdot[9 \mathrm{a}, 14 \mathrm{c}]$ The best fit values of $n$ for the $\mathrm{Cu}(111)$, $\mathrm{Cu}(653)^{R \& S}$ and $\mathrm{Cu}(643)^{R \& S}$ surfaces are $n$ $=10,8$ and 14 , respectively. The values of the free energies of homochiral aggregation were found to be $\sim 6.0 \mathrm{~kJ} / \mathrm{mol}$ (Asp/ $\mathrm{Cu}(111)), 3.3 \mathrm{~kJ} / \mathrm{mol}\left(\mathrm{Asp} / \mathrm{Cu}(653)^{R \& S}\right)$ and $2.2 \mathrm{~kJ} / \mathrm{mol}\left(\mathrm{Asp} / \mathrm{Cu}(643)^{R \& S}\right)$. The free energy of homochiral aggregation, $(\Delta G)$ was calculated using $\Delta G=R T \cdot \ln \left(K_{c}\right)$. Although we have no direct evidence of Asp aggregation on $\mathrm{Cu}$ surfaces, aggregation of adsorbates such as amino acids has been observed by STM on many surfaces. ${ }^{[14 a]}$

Fitting adsorption isotherms using the aggregation model described above can be used to determine the surface structure sensitivity of enantiomer aggregation. For example, it appears that surfaces with (111) terraces $\left(\mathrm{Cu}(653)^{R \& S}, \mathrm{Cu}(643)^{R \& S}\right.$ and $\mathrm{Cu}(111)$ ) tend to aggregate Asp homochirally. ${ }^{[9 a, 14 c]}$ On the other hand, the $\mathrm{Cu}(3,1,17)^{R \& S}$ surface with (100) terraces does not tend to aggregate amino acid enantiomers. Instead $\mathrm{Cu}(3,1,17)^{R \& S}$ adsorbs Asp enantiospecifically but shows no tendency for either aggregation or enantiospecificity for alanine, serine and phenylalanine.[7a,9b,13] While further studies need to be performed to determine which surface structural features cause aggregation

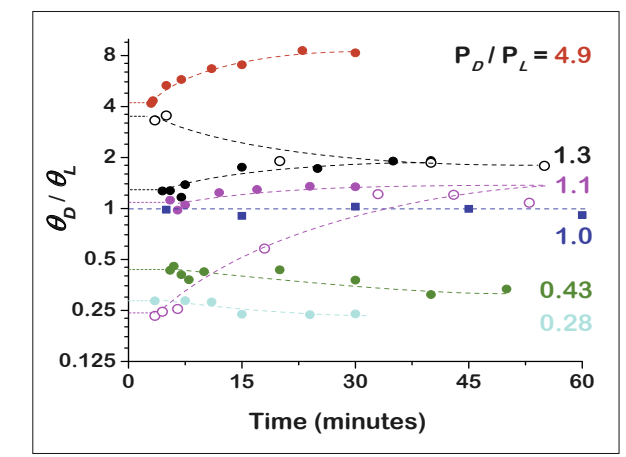




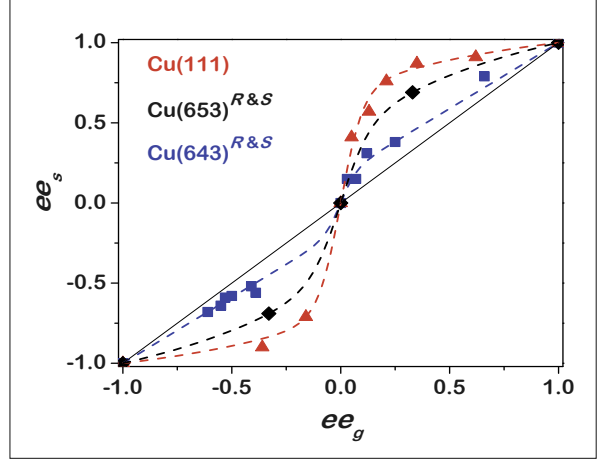

Fig. 7. Experimental and fit isotherms for enantiospecific adsorption of Asp/Cu(111) (red), Asp/Cu(653) ${ }^{R \& S}$ (black) and Asp/Cu(643) ${ }^{R \& S}$ (blue). All three exhibit homochiral enantiomer aggregation by amplification of enantiomer excess, $\left|e e_{s}\right|>\left|e e_{g}\right|$. However, there is no sign of enantiospecific adsorption; i.e. ee $e_{s}=0$ at $e e_{g}=0$.

for which amino acids, this work illustrates a method which can probe enantiospecific aggregation of enantiomers on chiral and achiral surfaces.

\section{Conclusion}

In this work, both enantiospecific decomposition kinetics and homochiral enantiomer aggregation have been identified on the naturally $\mathrm{Cu}(643)^{R \& S}$ surfaces. Enantiospecific isotopic labelling has enabled detailed analysis of the Asp decomposition mechanism on $\mathrm{Cu}(643)^{R \& S}$ demonstrating rigorously that Asp, adsorbed in its doubly deprotonated form, decomposes via cleavage of the $\mathrm{C} 3-\mathrm{C} 4$ bond, followed by cleavage of the $\mathrm{C} 1-\mathrm{C} 2$ bond and finally, the decomposition of the remaining $\mathrm{C}_{2} \mathrm{NH}_{5}$ fragment into $\mathrm{N} \equiv \mathrm{CCH}_{3}$ and $\mathrm{H}_{2}$. The combination of a naturally chiral surface coupled with a non-linear surface explosion reaction amplifies small enantiospecific differences in decomposition energetics into a factor of $\sim 6$ difference in enantiospecific decomposition rates on $\mathrm{Cu}(643)^{R \& S}$. Isotopic labeling also allows direct measurements of the adsorption-induced autoamplification of enantiomeric excess during equilibrium exposure of the $\mathrm{Cu}(643)^{R \& S}$ surfaces to non-racemic mixtures of Asp. Asp enantiomers have been shown to displace one another enantiospecifically on $\mathrm{Cu}(643)$ but, when equilibrated with $\mathrm{D}-/ \mathrm{L}-$ Asp mixtures in the gas phase, enantiospecific adsorption is suppressed in favor of homochiral aggregate formation.

\section{Acknowledgements}

This work has been funded by the US Department of Energy, Basic Energy Science program under grant number DE-SC0008703.

Received: April 9, 2018

[1] a) J. Gal, Helv. Chim. Acta 2013, 96, 1617; b) L. Pasteur, C. R. Acad. Sci. 1848, 26, 535.

[2] a) D. S. Sholl, A. J. Gellman, AIChE J. 2009 55, 2484, DOI: 10.1002/aic.12036; b) A. J. Gellman, W. T. Tysoe, F. Zaera, Catal. Lett. 2015, 145, 220, DOI: 10.1007/s10562-014 1400-8.

[3] a) M. Heitbaum, F. Glorius, I. Escher, Angew. Chem. Int. Ed. 2006, 45, 4732; b) D. Y. Murzin, P. Mäki-Arvela, E. Toukoniitty, T. Salmi, Catal. Rev. Sci. Eng. 2005, 47, 175; c) T. Mallat, E. Orglmeister, A. Baiker, Chem. Rev. 2007, 107 , 4863.

[4] a) A. R. Ribeiro, A. S. Maia, Q. B. Cass, M. E. Tiritan, J. Chromatogr. B 2014, 968, 8; b) Z. A. Al-Othman, A. Al-Warthan, S. D. Alam, I. Ali, Biomed. Chromatogr. 2014, 28, 1514; c) R. Sancho, C. Minguillon, Chem. Soc. Rev. 2009, 38, 797, DOI: 10.1039/b718359n; d) V. A. Soloshonok, C. Roussel, O. Kitagawa, A. E. Sorochinsky, Chem. Soc. Rev. 2012, 41, 4180, DOI: $10.1039 / \mathrm{c} 2 \mathrm{cs} 35006 \mathrm{~h}$.

[5] a) R. M. Hazen, D. S. Sholl, Nat. Mater. 2003, 2, 367; b) L. Addadi, S. Weiner, Nature 2001, 411,753 .

[6] a) J. D. Horvath, A. J. Gellman, Top. Catal. 2003, 25, 9; b) S. J. Jenkins, S. J. Pratt, Surf. Sci. Rep. 2007, 62, 373, DOI: 10.1016/j.surfrep.2007.06.001; c) C. F. McFadden, P. S. Cremer, A. J. Gellman, Langmuir 1996, 12 , 2483, DOI: $10.1021 / \mathrm{la} 9503481$.
[7] a) Y. Yun, A. J. Gellman, Langmuir 2015, 31, 6055, DOI: 10.1021/acs.langmuir.5b00707; b) A. J. Gellman, Y. Huang, A. J. Koritnik, J. D. Horvath, J. Phys.: Cond. Matt. 2017, 29 , 034001.

[8] a) J. D. Horvath, L. Baker, A. J. Gellman, J. Phys. Chem. C 2008, 112, 7637, DOI: 10.1021/ jp0753878; b) T. Greber, Z. Sljivancanin, R. Schillinger, J. Wider, B. Hammer, Phys. Rev. Lett. 2006, 96.

[9] a) Y. Yun, A. J. Gellman, J. Phys. Chem. C 2016, 120,27285 ; b) Y. Yun, D. Wei, D. S. Sholl, A. J. Gellman, J. Phys. Chem. C 2014, 118, 14957, DOI: $10.1021 /$ jp503796u

[10] J. Elemans, I. De Cat, H. Xu, S. De Feyter, Chem. Soc. Rev. 2009, 38, 722, DOI: 10.1039/ b800403j.

[11] a) R. Raval, Chem. Soc. Rev. 2009, 38, 707, DOI: $10.1039 / \mathrm{b} 800411 \mathrm{k}$; b) S. M. Barlow, R. Raval, Surf. Sci. Rep. 2003, 50, 201.

[12] B. S. Mhatre, S. Dutta, A. Reinicker, B Karagoz, A. J. Gellman, Chem. Comm. 2016, 52, 14125, DOI: 10.1039/c6cc06887a.

[13] Y. Yun, A. J. Gellman, Angew. Chem. Int. Ed. 2013, 52, 3394, DOI: 10.1002/anie. 201209025.

[14] a) S. Dutta, A. J. Gellman, Chem. Soc Rev. 2017, 46, 7787; b) A. J. Gellman, Y. Yun, Angew. Chem. Int. Ed. 2013, 52, 3394; c) A. J. Gellman, Y. Yun, Nat. Chem. 2015, 7, 520, DOI: 10.1038/nchem. 2250

[15] A. J. Gellman, Y. Huang, X. Feng, V. V. Pushkarev, B. Holsclaw, B. S. Mhatre, J. Am. Chem. Soc. 2013, 135, 19208.

[16] R. M. Hazen, T. R. Filley, G. A. Goodfriend, PNAS 2001, 98, 5487.

[17] J. Jacques, A. Collet, S. H. Wilen, 'Enantiomers, racemates, and resolutions', Wiley, 1981.

[18] a) J. McCarty, J. Falconer, R. J. Madix, J. Catal. 1973, 30, 235; b) J. Falconer, R. Madix, Surf. Sci. 1974, 46, 473; c) J. Falconer, J. McCarty, R. Madix, Japan. J. Appl. Phys. 1974, 13, 525.

[19] a) M. O. Lorenzo, V. Humblot, P. Murray, C. J. Baddeley, S. Haq, R. Raval, J. Catal. 2002, 205, 123, DOI: 10.1006/jcat.2001.3422; b) B. Behzadi, S. Romer, R. Fasel, K. H. Ernst, J. Am. Chem. Soc. 2004, 126, 9176, DOI: 10.1021/ ja048206d; c) B. S. Mhatre, V. Pushkarev, B. Holsclaw, T. J. Lawton, E. C. H. Sykes, A. J. Gellman, J. Phys. Chem. C 2013, 117, 7577, DOI: $10.1021 /$ jp3119378.

[20] a) J. D. Horvath, A. Koritnik, P. Kamakoti, D. S. Sholl, A. J. Gellman, J. Am. Chem. Soc. 2004, 126, 14988, DOI: 10.1021/ja45537h; b) Y. Huang, A. J. Gellman, Top. Catal. 2011, 54, 1403, DOI: 10.1007/s11244-011-9756-0. 